\title{
Augmentation of Mind-body Therapy and Role of Deep Slow Breathing
}

ARTICLE in JOURNAL OF COMPLEMENTARY AND INTEGRATIVE MEDICINE · JANUARY 2009

DOI: $10.2202 / 1553-3840.1299$

CITATIONS

4

2 AUTHORS:

Ravinder Jerath

Augusta Women's Center

16 PUBLICATIONS 130 CITATIONS

SEE PROFILE

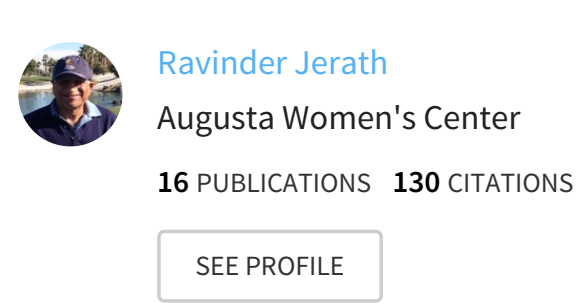

READS

150

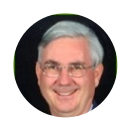

Vernon A Barnes

Georgia Regents University

53 PUBLICATIONS 1,009 CITATIONS

SEE PROFILE 
An Article Submitted to

\title{
Journal of Complementary and Integrative Medicine
}

Manuscript 1299

\section{Augmentation of Mind-body Therapy and Role of Deep Slow Breathing}

\author{
Ravinder Jerath* $^{*} \quad$ Vernon A. Barnes ${ }^{\dagger}$
}

*Augusta Women's Center, rj605r@aol.com

†Medical College of Georgia, vbarnes@ mail.mcg.edu

Copyright (c)2009 The Berkeley Electronic Press. All rights reserved. 


\title{
Augmentation of Mind-body Therapy and Role of Deep Slow Breathing*
}

\author{
Ravinder Jerath and Vernon A. Barnes
}

\begin{abstract}
Mind-body therapies have been shown to be effective in clinical treatment of disorders such as high blood pressure and stress. Significant differences in the effectiveness of mind-body therapies have been shown and a common link among the therapies has yet to be defined. This article overviews the role of slow rhythmic breathing in physiological as well as therapeutic effects of mind-body therapies. Slow deep breathing practice has important implications as it may underlie the basic mechanism that synchronizes the brain with the autonomic response. This article reviews studies that include the effect of deep slow breathing with or without mind-body therapy exercises. In utero studies that monitor patterns of fetal breathing reveal sympathetic activation with irregular, shallow fast breathing movements compared to slow deep breathing. Recognition of respiratory mechanisms in mind-body therapies can lead to development of more effective relaxation exercises that may incorporate deep slow breathing in clinical applications.
\end{abstract}

KEYWORDS: breathing, meditation, hypertension, stress

*Please send correspondence to Ravinder Jerath, M.D., Augusta Women's Center, Suite 6\&7, 2100 Central Avenue, Augusta, Georgia 30904. Telephone: 706-736-5378. Fax: 706-738-9922. E-mail: RJ605R@aol.com. 
Jerath and Barnes: Role of Deep Slow Breathing in Mind-body Therapy

\section{Introduction}

In a report on the nature and psychological problems in general practice, $54 \%$ of females and $46 \%$ of male patients had experienced some level of psychological problems. The most common disorders were depression, anxiety and substance abuse disorders (MaGPIe Research Group, 2003). In a World Health Organization study at 15 international sites, $24 \%$ frequency of psychological disorders in primary care settings was noted (Sartorius et al., 1996)

Psychological stress has been thought to play a significant role in the development of hypertension by acting on the autonomic nervous system (Treiber et al., 2001; Snieder et al., 2002). Chronic activation of the sympathetic nervous system has been linked to essential hypertension (Esler, 2000). The acute phase of the stress response leads to increased sympathetic activation (Julius and Majahalme, 2000). Animal and human studies have demonstrated that exposure to acute and chronic stress may augment sympathetic nervous system activation resulting in hypertension (Guyton and Hall, 2005). The breathing pattern has been found to be the underlying mechanism for the sympathetic or parasympathetic response, for example, increased fast irregular breathing is associated with an increased sympathetic response (Eckberg, 2000).

\section{Effects of Deep Slow Breathing on Blood Pressure}

Applying rules of evidence-based medicine, two series of systematic reviews of mind-body therapies were compared where it was noted that relaxation therapy is beneficial for anxiety and insomnia and hypnotherapy is useful for pain (Ernst et al., 2007). In a study where relaxation techniques (e.g., music therapy, autogenic training, biofeedback) were compared to imagery training, the relaxation techniques were associated with the parasympathetic response and a slow breathing pattern, whereas such a response was not noticed with imagery (Blumenstein et al., 1995). Slow deep breathing as practiced in various mind-body therapies is associated with the parasympathetic response (Jerath et al., 2006). Monitored relaxation with biofeedback-assisted deep-diaphragmatic breathing, revealed a breathing pattern similar to meditation (Fried, 1987). Monitoring of brainstem neurons during slow breathing revealed $<0.15-\mathrm{Hz}$ band cardiovascular rhythms, suggested to be a common origin of identical phenomena in humans and animals in the reticular formation of the brain stem (Perlitz et al., 2004). In a study comparing the cardio-respiratory phase synchronization between inwardattention meditation and normal relaxation, the rhythm-ratio synchronization between heart rate and respiratory rate was noted to be 4:1 and 5:1. The study showed weak cardio-respiratory phase synchronization in normal relaxation compared to it being enhanced in meditation (Wu and Lo, 2009). In a similar 
study, a group of practitioners of Zen meditation revealed enhanced parasympathetic response when a slow breathing $<0.15 \mathrm{~Hz}$ frequency was practiced (Wu and Lo, 2008).

\section{Augmented Response from Breathing on Hypertension}

Fetal breathing movements are known to influence blood pressure and heart rate. Irregular shallow fast breathing in the fetus is associated with increase in blood pressure and heart rate (Cosmi et al., 2001). Normotensive middle-school children (Barnes et al., 2004) and pre-hypertensive adolescents (Barnes et al., 2008) evaluated for ambulatory systolic blood pressure changes showed significant greater blood pressure reduction with breathing awareness meditation. This type of meditation does not involve manipulation of the breath. Similar blood pressure decreases in pre-hypertensive adolescents were reported in a similar study with Transcendental Meditation ${ }^{\circledR}$ (TM) (Barnes et al., 2004). A recent meta-analysis that compared well-controlled randomized trials in modulating the blood pressure revealed that TM is more effective than biofeedback, progressive muscle relaxation or stress management training (Rainforth et al., 2007). No specific breath control is involved in the practice of the TM technique, however, a reduction of respiration rate has been reported (Dillbeck and Orme-Johnson, 1987).

A large study of 21,565 subjects practicing deep breathing for 30 seconds induced significant blood pressure decreases in normotensive, untreated and treated hypertensives (Mori et al., 2005). When slow deep breathing is practiced for 5 minutes applying the pranayama technique (an alternate nostril slow deep breathing technique), a significant reduction in both systolic and diastolic blood pressure was noted immediately after the exercise (Pramanik et al., 2009).

In patients with resistant hypertension, device-guided slow deep breathing without meditation showed a significant reduction in ambulatory and home blood pressure (Grossman et al., 2001; Viskoper et al., 2003; Meles et al., 2004). Slow deep breathing respiratory exercise (1 breath per minute) for 30 minutes daily on hemodynamic variables indicated dramatic shifts of hemodynamic variables that were suggestive of preventing or eliminating heart attacks. It was postulated that this effect is associated with resetting the cardio-respiratory brain-stem pacemaker (Shannahoff-Khalsa et al., 2004).

Decrease in incidence of pre-eclampsia and improvement of hypertension has been indicated with relaxation exercises and deep slow breathing (Jerath et al., 2009). A line of evidence supporting this theory is the decrease in the incidence of pre-eclampsia following the restoration of normal breathing. Induced maternal relaxation during the 32nd week of pregnancy resulted in significant changes in maternal heart rate, skin conduction and increased respiratory sinus arrhythmia. 
Parallel significant alterations of fetal neural behavior were observed including decreased fetal heart rate, and increased fetal heart rate variability with increased fetal movement-heart rate coupling (DiPietro et al., 2008). Studies conducted with pregnant patients with abnormal umbilical artery Doppler findings demonstrated significantly improved blood flow when yoga was practiced with deep slow breathing techniques (Narendran et al., 2005). A case-controlled prospective study of pregnant women who practiced a slow, deep breathing-based yoga technique starting at 18-20 weeks showed significantly decreased incidence of pre-term birth and pre-eclampsia (Narendran et al., 2005).

\section{Effects of Deep Slow Breathing on Stress}

Oxidative stress may contribute to pathophysiology of many chronic diseases such as psychosocial stress. A study using stress reduction from Sudarshan Kriya (deep slow breathing meditation) revealed significantly lower levels of lactate, and higher levels of superoxidase dismutase, glutathione and catalase compared with controls (Sharma et al., 2003). In another study, hemodynamic response to acute stress to healthy volunteers revealed significantly positive effect when slow deep breathing technique was applied (Nogawa et al., 2007). After a two-month $\mathrm{TM}$ intervention, greater decreases in SBP reactivity to laboratory stress were observed compared controls (Barnes et al., 2001).

Treatment with slow deep breathing in climacteric symptoms has been shown to decrease stress and neuroticism in women. A randomized controlled study of one hundred twenty five participants (ages 40-55) practicing yoga with pranayama showed a decrease in perceived stress and neuroticism compared to controls (Chattha et al., 2008). A randomized controlled trial of pranayama with selected yoga asanas (postures) and slow deep breathing practiced for 3 months resulted in significant improvement in cardio-respiratory performance, psychological profile and an increase in plasma melatonin level. The maximum night time melatonin levels in the yoga group showed significant correlation with well-being (Harinath et al., 2004). Patients with obsessive-compulsive disorder who practiced Kundalini yoga meditation were compared with the relaxation response plus mindfulness meditation. At 3 months, there was significant improvement in the obsessive compulsive scale, global severity index, profile of mood scales, and suggests that Kundalini yoga techniques are effective in the treatment of obsessive-compulsive disorder (Shannahoff-Khalsa et al., 1999).

\section{Conclusion}

In utero, when irregular, fast shallow breathing is observed in the fetus, it is associated with the sympathetic response. Brainstem monitoring in humans and 
animals demonstrates synchronization of the cardio-respiratory system at the frequency of 6 breaths per minute. Deep slow breathing techniques lead to positive autonomic balance that affects brain, heart, and other organ systems innervated by the autonomic nervous system. It leads to drop in blood pressure and heart rate even without any meditative practice. However, when combined with a meditative practice the effects of slow deep breathing are noted to be significantly greater for hypertension, stress and anxiety, obsessive-compulsive disorder, and climacteric symptoms of post-menopausal women compared other relaxation techniques. Augmentation of the physiologic response from slow deep breathing appears to stem from resetting of the brain stem cardio-respiratory center. Controlled studies have shown significantly improved physiological, psychological and clinical response. Further studies are needed to compare techniques with and without deep slow breathing to validate its clinical benefit. In combination with other relaxation methods, deep slow breathing techniques may produce a better outcome in prevention and treatment of hypertension, preeclampsia, heart and psychological diseases.

\section{References}

Barnes, V. A., H. C. Davis, J. B. Murzynowski and F. A. Treiber (2004). Impact of meditation on resting and ambulatory blood pressure and heart rate in youth. Psychosom Med 66(6): 909-14.

Barnes, V. A., M. H. Johnson and F. A. Treiber (2004). Impact of Transcendental Meditation on ambulatory blood pressure in African American adolescents. Am J Hypertens 17(4): 366-69.

Barnes, V. A., R. A. Pendergrast, G. A. Harshfield and F. A. Treiber (2008). Impact of meditation on ambulatory blood pressure and sodium handling in prehypertensive African American adolescents. Ethn Dis 18(1): 1-5.

Barnes, V. A., F. A. Treiber and H. Davis (2001). Impact of Transcendental Meditation on cardiovascular function at rest and during acute stress in adolescents with high normal blood pressure. J Psychosom Res 51(4): 597-605.

Blumenstein, B., I. Breslav, M. Bar-Eli, G. Tenenbaum and Y. Weinstein (1995). Regulation of mental states and biofeedback techniques: effects on breathing pattern. Biofeedback Self Regul 20(2): 169-83.

Chattha, R., N. Raghuram, P. Venkatram and N. R. Hongasandra (2008). Treating the climacteric symptoms in Indian women with an integrated approach to yoga therapy: a randomized control study. Menopause 15(5): 862-70.

Cosmi, E. V., E. Cosmi and R. La Torre (2001). The effects of fetal breathing movements on the utero-fetal-placental circulation. Early Pregnancy 5(1): $51-2$. 
Dillbeck, M. C. and D. W. Orme-Johnson (1987). Physiological differences between Transcendental Meditation and rest. American Psychologist 42: 879-81.

DiPietro, J. A., K. A. Costigan, P. Nelson, E. Gurewitsch and M. L. Laudenslager (2008). Fetal responses to induced maternal relaxation during pregnancy. Biol Psychol 77(1): 11-19.

Eckberg, D. L. (2000). Physiological basis for human autonomic rhythms. Ann Med 32(5): 341-9.

Ernst, E., M. H. Pittler, B. Wider and K. Boddy (2007). Mind-body therapies: are the trial data getting stronger? Altern Ther Health Med 13(5): 62-4.

Esler, M. (2000). The sympathetic system and hypertension. Am J Hypertens $13(6$ Pt 2): 99S-105S.

Fried, R. (1987). Relaxation with biofeedback-assisted guided imagery: the importance of breathing rate as an index of hypoarousal. Biofeedback Self Regul 12(4): 273-9.

Grossman, E., A. Grossman, M. H. Schein, R. Zimlichman and B. Gavish (2001). Breathing-control lowers blood pressure. J Hum Hypertens 15(4): 263-9.

Guyton, A. C. and J. E. Hall (2005). Textbook of Medical Physiology. Philadelphia, W.B. Saunders Company.

Harinath, K., A. S. Malhotra, K. Pal, R. Prasad, R. Kumar, T. C. Kain, L. Rai and R. C. Sawhney (2004). Effects of Hatha yoga and Omkar meditation on cardiorespiratory performance, psychologic profile, and melatonin secretion. J Altern Complement Med 10(2): 261-8.

Jerath, R., V. A. Barnes and H. E. Fadel (2009). Mechanism of development of pre-eclampsia linking breathing disorders to endothelial dysfunction. Med Hypotheses 73(2): 163-6.

Jerath, R., J. W. Edry, V. A. Barnes and V. Jerath (2006). Physiology of long pranayamic breathing: Neural respiratory elements may provide a mechanism that explains how slow deep breathing shifts the autonomic nervous system. Med Hypotheses 67(3): 566-71.

Julius, S. and S. Majahalme (2000). The changing face of sympathetic overactivity in hypertension. Ann Med 32(5): 365-70.

MaGPIe Research Group (2003). The nature and prevalence of psychological problems in New Zealand primary healthcare: a report on Mental Health and General Practice Investigation (MaGPIe). N Z Med J 116(1171): U379

Meles, E., C. Giannattasio, M. Failla, G. Gentile, A. Capra and G. Mancia (2004). Nonpharmacologic treatment of hypertension by respiratory exercise in the home setting. Am J Hypertens 17(4): 370-4. 
Mori, H., H. Yamamoto, M. Kuwashima, S. Saito, H. Ukai, K. Hirao, M. Yamauchi and S. Umemura (2005). How does deep breathing affect office blood pressure and pulse rate? Hypertens Res 28(6): 499-504.

Narendran, S., R. Nagarathna, S. Gunasheela and H. R. Nagendra (2005). Efficacy of yoga in pregnant women with abnormal Doppler study of umbilical and uterine arteries. J Indian Med Assoc 103(1): 12-17.

Narendran, S., R. Nagarathna, V. Narendran, S. Gunasheela and H. R. Nagendra (2005). Efficacy of yoga on pregnancy outcome. J Altern Complement Med 11(2): 237-44.

Nogawa, M., T. Yamakoshi, A. Ikarashi, S. Tanaka and K. Yamakoshi (2007). Assessment of slow-breathing relaxation technique in acute stressful tasks using a multipurpose non-invasive beat-by-beat cardiovascular monitoring system. Conf Proc IEEE Eng Med Biol Soc: 5323-5.

Perlitz, V., M. Lambertz, B. Cotuk, R. Grebe, R. Vandenhouten, G. Flatten, E. Petzold, H. Schmid-Schönbein and P. Langhorst (2004). Cardiovascular rhythms in the $0.15-\mathrm{Hz}$ band: common origin of identical phenomena in man and dog in the reticular formation of the brain stem? Pflugers Arch 448(6): 579-91.

Pramanik, T., H. O. Sharma, S. Mishra, A. Mishra, R. Prajapati and S. Singh (2009). Immediate effect of slow pace bhastrika pranayama on blood pressure and heart rate. J Altern Complement Med 15(3): 293-5.

Rainforth, M. V., R. H. Schneider, S. I. Nidich, C. Gaylord-King, J. Salerno and J. W. Anderson (2007). Stress reduction programs in patients with elevated blood pressure: A systematic review and meta-analysis. Curr Hypertens Rep 9: 520-28.

Sartorius, N., T. B. Ustün, Y. Lecrubier and H. Wittchen (1996). Depression comorbid with anxiety: results from the WHO study on psychological disorders in primary health care. Br J Psychiatry Suppl 30: 38-43.

Shannahoff-Khalsa, D. S., L. E. Ray, S. Levine, C. C. Gallen, B. J. Schwartz and J. J. Sidorowich (1999). Randomized controlled trial of yogic meditation techniques for patients with obsessive-compulsive disorder. CNS Spectr 4(12): 34-47.

Shannahoff-Khalsa, D. S., B. B. Sramek, M. B. Kennel and S. W. Jamieson (2004). Hemodynamic observations on a yogic breathing technique claimed to help eliminate and prevent heart attacks: a pilot study. J Altern Complement Med 10(5): 757-66.

Sharma, H., S. Sen, A. Singh, N. K. Bhardwaj, V. Kochupillai and N. Singh (2003). Sudarshan Kriya practitioners exhibit better antioxidant status and lower blood lactate levels. Biol Psychol 63(3): 281-91. 
Snieder, H., G. Harshfield, P. Barbeau, D. Pollock, J. Pollock and F. Treiber (2002). Dissecting the genetic architecture of the cardiovascular and renal stress response. Biol Psychol 61(1-2): 73-95.

Treiber, F. A., H. Davis and J. R. Turner (2001). Cardiovascular responsivity to stress and preclinical manifestations of cardiovascular disease in youth. Health and Behavior in Childhood and Adolescence: Cross-disciplinary Perspectives. L. Hayman, M. McMahon and J. R. Turner. New York, Lawrence Erlbaum Associates, Inc.

Viskoper, R., I. Shapira, R. Priluck, R. Mindlin, L. Chornia, A. Laszt, D. Dicker, B. Gavish and A. Alter (2003). Nonpharmacologic treatment of resistant hypertensives by device-guided slow breathing exercises. Am J Hypertens 16(6): 484-7.

$\mathrm{Wu}, \mathrm{S}$. D. and P. C. Lo (2008). Inward-attention meditation increases parasympathetic activity: a study based on heart rate variability. Biomed Res 29(5): 245-50.

Wu, S. D. and P. C. Lo (2009). Cardiorespiratory phase synchronization during normal rest and inward-attention meditation. Int J Cardiol. 